Article

\title{
Sensitivity Enhancement in Surface Plasmon Resonance Biochemical Sensor Based on Transition Metal Dichalcogenides/Graphene Heterostructure
}

\author{
Xiang Zhao ${ }^{1}$, Tianye Huang ${ }^{1, * \mathbb{C}}$, Perry Shum Ping ${ }^{2}, \mathrm{Xu} \mathrm{Wu}^{1}$, Pan Huang ${ }^{1}$, Jianxing Pan ${ }^{1}$, \\ Yiheng $\mathrm{Wu}^{1}$ and Zhuo Cheng ${ }^{1}$ \\ 1 School of Mechanical Engineering and Electronic Information, China University of Geosciences (Wuhan), \\ Wuhan 430074, China; zhaoxiang@cug.edu.cn (X.Z.); wuxu101393@cug.edu.cn (X.W.); \\ huang_pan@cug.edu.cn (P.H.); jianxing_pan@cug.edu.cn (J.P.); wuyiheng@cug.edu.cn (Y.W.); \\ chengzhuo@cug.edu.cn (Z.C.) \\ 2 Center of Fiber Technology, School of Electrical and Electronic Engineering, Nanyang Technological \\ University, Singapore 639798, Singapore; EPShum@ntu.edu.sg \\ * Correspondence: Tianye_huang@163.com or huangty@cug.edu.cn
}

Received: 14 May 2018; Accepted: 21 June 2018; Published: 27 June 2018

\begin{abstract}
In this work, a surface plasmon resonance (SPR) biosensor based on two-dimensional transition metal dichalcogenides (TMDCs) is proposed to improve the biosensor's sensitivity. In this sensor, different kinds of two-dimensional TMDCs are coated on both surfaces of metal film. By optimizing the structural parameters, the angular sensitivity can reach as high as $315.5 \mathrm{Deg} / \mathrm{RIU}$ with 7-layers $\mathrm{WS}_{2}$ and $36 \mathrm{~nm} \mathrm{Al}$ thin film, which is 3.3 times of the conventional structure based on single Al thin film. We also obtain maximum phase sensitivity $\left(3.85 \times 10^{6} \mathrm{Deg} / \mathrm{RIU}\right)$ with bilayer $\mathrm{WS}_{2}$ and $35 \mathrm{~nm} \mathrm{Al}$ thin film. The phase sensitivity can be further improved by employing $\mathrm{Ag}$ and removing air layer. The proposed configuration is of great potential for biochemical sensing.
\end{abstract}

Keywords: surface plasmon resonance; transition metal dichalcogenides; angular sensitivity; differential phase

\section{Introduction}

Surface plasmon resonance (SPR) is an optical phenomenon which occurs at the metal-dielectric surface. When light reflects at a SPR angle, free electrons on the metal surface can resonate and absorb light energy, consequently leading to a drastic attenuation of reflected light [1,2]. The SPR condition is sensitive to the environment variations and can be utilized as sensors. The biological molecules interactions in the sensing medium are detected by observing the refractive index changes of the sensor region. Due to advantages such as convenient detection, high sensitivity, real-time measurement, SPR sensors have been used to detect and analyze various biological molecules, such as proteins, nucleic acids and viruses, and have a broad prospect in practical applications [3-6]. Sensitivity is one of the most important aspects for biological sensing in particular, and how to enhance the sensitivity becomes a research hotspot for SPR biosensors.

Recently, 2D materials such as graphene and transition metal dichalcogenides (TMDCs) have are well-known for their use in constructing SPR sensors due to their unique electrical and optical properties [7]. This is because firstly, the high real part of the dielectric constant allows them to help metal absorb light energy [8]. Secondly, some features such as high surface to volume ratio and tunable biocompatibility can help the biosensor obtain sensitivity enhancements [9]. Finally, when coating these materials on the metal film, they can also protect the metal from oxidation as protective layers $[10,11]$. Based on these advantages, various 2D-material-assisted SPR sensors are proposed and investigated. 
Graphene has been proposed for the enhancement of the sensitivity [12]. Zeng et al. presented a highly sensitive SPR biosensor based on graphene- $\mathrm{MoS}_{2}$ hybrid nanostructures to enhance its sensitivity [13]. Air layer and graphene sheet for sensitivity enhancement was analyzed in [14]. Other TMDCs like $\mathrm{WS}_{2}, \mathrm{MoSe}_{2}$ and $\mathrm{WSe}_{2}$ are combined with silicon to enhance the sensitivity [15]. Wu et al. proposed a SPR biochemical sensor with heterostructures of few-layer BP and 2D materials (graphene $/ \mathrm{MoS}_{2} / \mathrm{WS}_{2} / \mathrm{MoSe}_{2} / \mathrm{WSe}_{2}$ ) [16]. According to the previous work, it is found that the sensor performances are highly related to the structures and functional materials. To further enhance the sensitivity, both of these two aspects should be properly optimized.

In this paper, SPR sensor constructed by TMDCs/metal/TMDCs/graphene heterostructure is used for both angular and phase sensitivity enhancement. By coating different 2D TMDCs $\left(\mathrm{MoS}_{2} / \mathrm{MoSe}_{2} / \mathrm{WS}_{2} / \mathrm{WSe}_{2}\right)$ at both sides of the metal, the sensitivity of the proposed sensor can be improved by the enhancement of light-material interaction. Angular sensitivity as high as $315.5 \mathrm{Deg} / \mathrm{RIU}$ which is nearly 3 times that of conventional configurations can be obtained. Furthermore, the proposed SPR configuration is suitable for phase detection as well and a pronounced phase sensitivity up to $3.85 \times 10^{6} \mathrm{Deg} / \mathrm{RIU}$ is predicted.

\section{Sensor Configuration and Theoretical Model}

The schematic diagram of the proposed SPR biosensor is shown in Figure 1a, the configuration contains seven layers and the operation wavelength is $633 \mathrm{~nm}$ which is popular for SPR applications $[13,14,17]$. BK7 glass with refractive index of $n_{\mathrm{p}}=1.5151$ acts as the coupling prism [18]. The refractive index of the air layer is fixed at 1 with thickness of $35 \mathrm{~nm}$. The metal employed in this configuration is $\mathrm{Al}$ with dielectric constant of $-34.2574+0.9108 \mathrm{i}$ [19]. Various TMDCs, represented by $\mathrm{MX}_{2}$, are coated at both sides of $\mathrm{Al}$ thin film, the thickness and refractive index of TMDCs at $633 \mathrm{~nm}$ are shown in Table $1[20,21]$. The graphene layer is coated on the $\mathrm{MX}_{2} / \mathrm{Al} / \mathrm{MX}_{2}$ hybrid structure as the biomolecular recognition element and the refractive index of graphene is given as [22]:

$$
n_{G}=3.0+\frac{i C_{1} \lambda}{3}
$$

where $\lambda$ is the wavelength and $C_{1}=5.446 \mu \mathrm{m}^{-1}$. The thickness of the monolayer graphene is $0.34 \mathrm{~nm}$. The refractive index of the sensing medium is given as $n_{\mathrm{s}}=1.33+\Delta n$, where $\Delta n$ is the index change of the sensing medium.

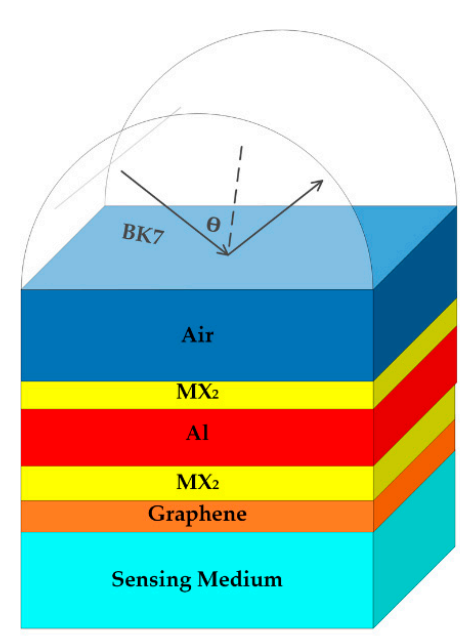

(a)

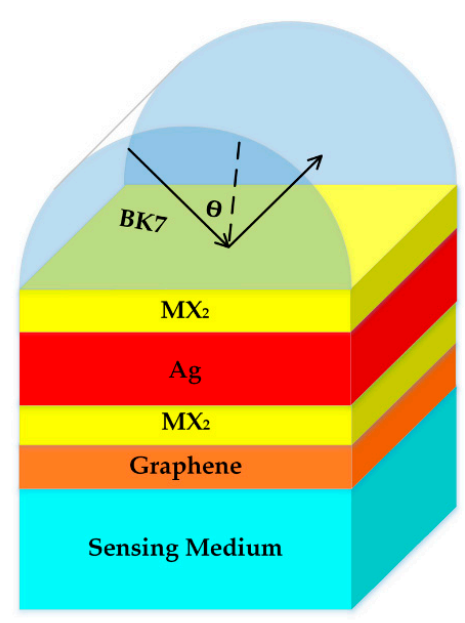

(b)

Figure 1. Schematic diagram of the SPR biosensor for (a) angle-sensitivity enhancement and (b) phase-sensitivity enhancement. 
Table 1. The thickness, refractive index and optical constants of different $M X_{2}$ at $\lambda=633 \mathrm{~nm}$.

\begin{tabular}{cccc}
\hline Type of TMDC & Thickness of Monolayer (nm) & Refractive Index & Dielectric Constant \\
\hline $\mathrm{MoS}_{2}$ & 0.65 & $5.0805+1.1723 \mathrm{i}$ & $24.4368+11.9121 \mathrm{i}$ \\
$\mathrm{MoSe}_{2}$ & 0.70 & $4.6226+1.0063 \mathrm{i}$ & $20.3560+9.3039 \mathrm{i}$ \\
$\mathrm{WS}_{2}$ & 0.80 & $4.8937+0.3124 \mathrm{i}$ & $23.8511+3.0578 \mathrm{i}$ \\
$\mathrm{WSe}_{2}$ & 0.70 & $4.5501+0.4332 \mathrm{i}$ & $20.5156+3.9423 \mathrm{i}$ \\
\hline
\end{tabular}

In this paper, for the SPR curve calculation and sensing performance analysis, the transfer matrix method (TMM) is employed [23]. In the proposed structure, the thickness, the refractive index, and the dielectric constant of each layer are defined as $d_{\mathrm{k}}, n_{\mathrm{k}}$ and $\varepsilon_{\mathrm{k}}$, respectively. The incident angle corresponding to the minimum reflectance is called resonance angle and the angular sensitivity is calculated by probing the spectral shifts of the resonance angle [3] and defined as $S_{\mathrm{A}}=\Delta \theta_{\text {res }} / \Delta n$ [24], where $\Delta \theta_{\text {res }}$ represents the change of resonance angle. Furthermore, we also discuss phase sensitivity which is defined as $S_{\mathrm{p}}=\Delta \varphi / \Delta n$ [13], where $\Delta \varphi$ is the differential phase changes corresponding to $\Delta n$.

\section{Results and Discussions}

In order to obtain the optimal angular sensitivity for the proposed configuration, we firstly calculate the angular sensitivity with various number of $\mathrm{MX}_{2}$ layers and $\mathrm{Al}$ thickness. It should be noted that in this calculation, the $\mathrm{MX}_{2}$ layers at both sides are changed simultaneously. As shown in Figure $2 \mathrm{a}-\mathrm{d}$, when the refractive index of sensing medium changes from 1.330 to $1.335(\Delta n=0.005)$, the SPR curves shows three important features enumerated below.

(1) When the thickness of $\mathrm{Al}$ thin film is fixed, the sensitivity increases with more $\mathrm{MX}_{2}$ layers mainly due to the enhanced light energy absorption. However, it will decrease rapidly when the number of $\mathrm{MX}_{2}$ layers exceeds the optimal number which is defined as the number of $\mathrm{MX}_{2}$ layers with the highest sensitivity.

(2) The optimal numbers of $\mathrm{MX}_{2}$ layers will increase when the thickness of $\mathrm{Al}$ increases.

(3) With the same thickness of $\mathrm{Al}$ thin film, the enhancement effect offered by different kinds of $\mathrm{MX}_{2}$ are not the same.

It is known that the improvement effect caused by TMDCs is related to their dielectric constants. As illustrated in Table 1, MoS 2 has a larger real part of the dielectric constant than others, which means its absorption ability is stronger [15]. Nevertheless, the ability to absorb light is not the only crucial factor to affect sensitivity; electron energy loss related to the imaginary part of the dielectric constant can lead to a counteraction [13]. Comparing to other TMDCs, the $\mathrm{WS}_{2}$ layers have much lower energy loss because of their small imaginary part of the dielectric constant. According to the conditions above, the optimized parameters and the corresponding angular sensitivity for different TMDCs are summarized in Table 2. The highest sensitivities can achieve 214.8 Deg/RIU, 210.1 Deg/RIU, 315.5 Deg/RIU and 286.3 Deg/RIU for the structures containing 3-layer $\mathrm{MoS}_{2}$ with $22 \mathrm{~nm} \mathrm{Al} \mathrm{film,}$ 4-layer $\mathrm{MoSe}_{2}$ with $24 \mathrm{~nm} \mathrm{Al} \mathrm{film,} \mathrm{7-layer} \mathrm{WS}_{2}$ with $36 \mathrm{~nm} \mathrm{Al} \mathrm{film} \mathrm{and} \mathrm{7-layer} \mathrm{WSe} \mathrm{W}_{2}$ with $30 \mathrm{~nm} \mathrm{Al}$ film, respectively. The corresponding SPR curves are shown in Figure 2e-h. 


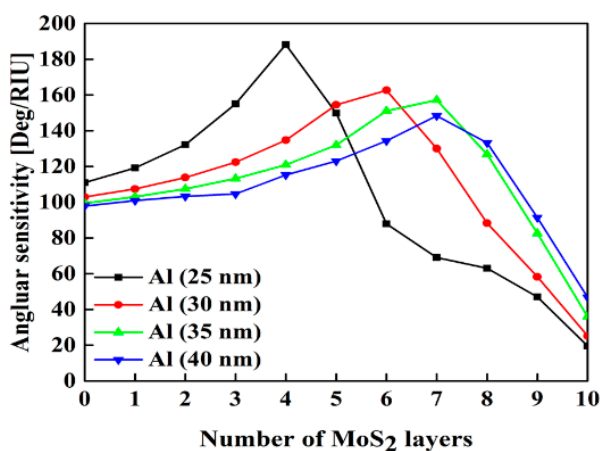

(a)

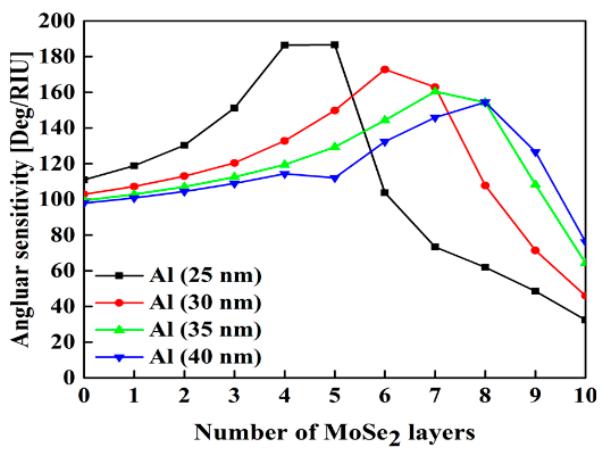

(b)

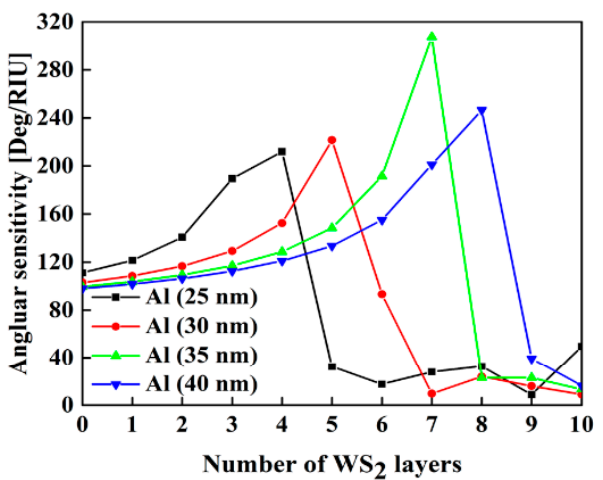

(c)

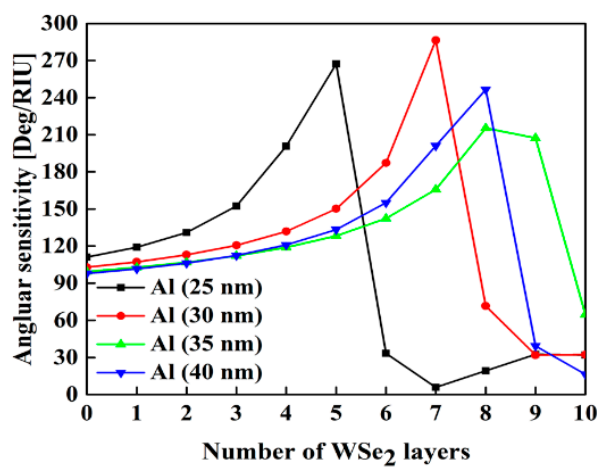

(d)

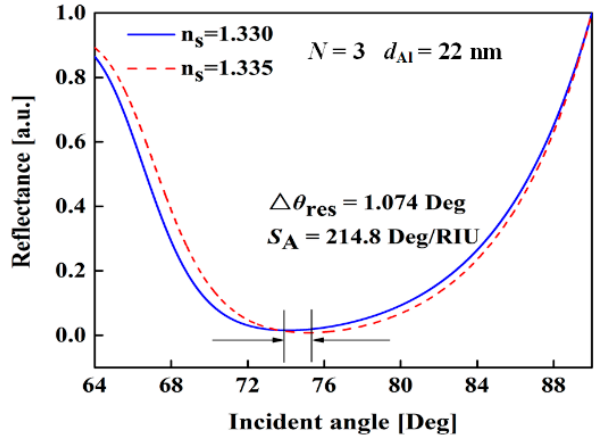

(e)

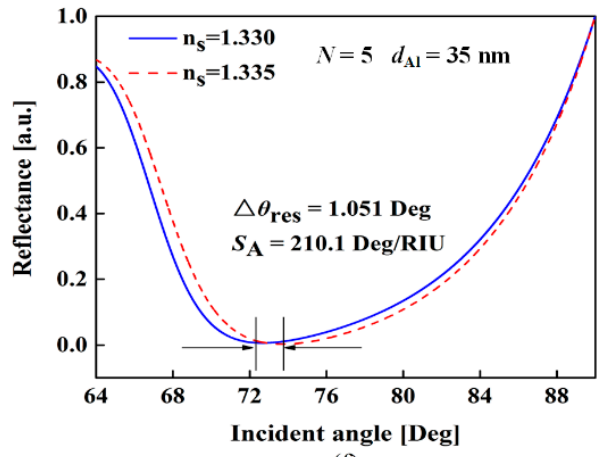

(f)

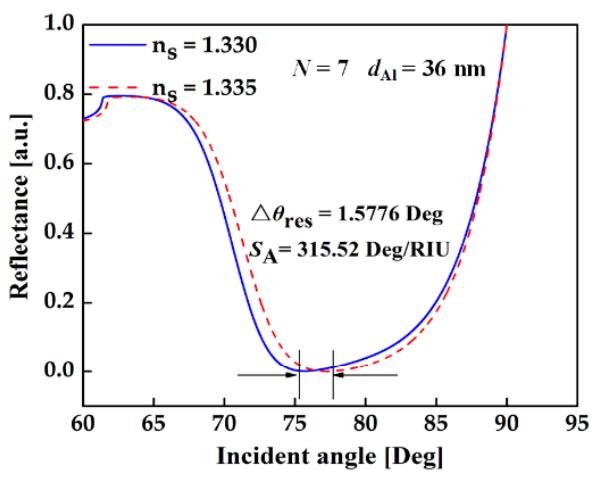

(g)

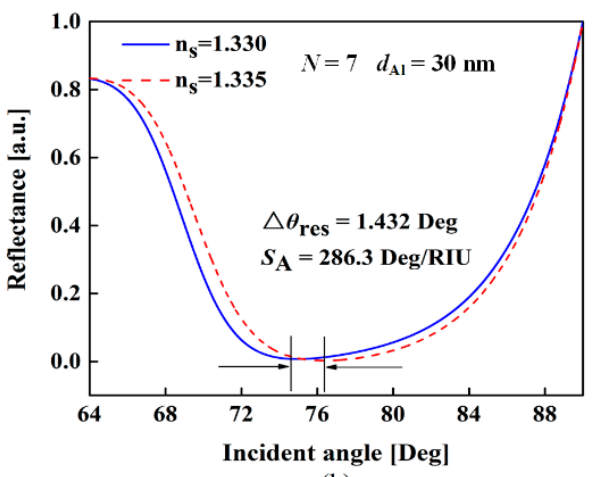

(h)

Figure 2. (a-d) Variation of the angular sensitivity as a function of the number of $M X_{2}$ layers $N$ with various thickness of $\mathrm{Al}$ thin film $d_{\mathrm{Al}}$ and $(\mathbf{e}-\mathbf{h})$ the reflection spectra with different TMDCs materials under optimal structures. 
Table 2. Optimized values of thickness of gold and the number of $\mathrm{MoS}_{2}$ layers with corresponding change in angular sensitivity.

\begin{tabular}{cccc}
\hline Type of TMDC & Optimal Thickness of Al (nm) & Optimal Number of TMDC Layers & Angular Sensitivity $(\boldsymbol{\Delta} \boldsymbol{n}=\mathbf{0 . 0 0 5})$ \\
\hline $\mathrm{MoS}_{2}$ & 22 & 3 & $214.8 \mathrm{Deg} / \mathrm{RIU}$ \\
$\mathrm{MoSe}_{2}$ & 24 & 4 & $210.1 \mathrm{Deg} / \mathrm{RIU}$ \\
$\mathrm{WS}_{2}$ & 36 & 7 & $315.5 \mathrm{Deg} / \mathrm{RIU}$ \\
$\mathrm{WSe}_{2}$ & 30 & 7 & $286.3 \mathrm{Deg} / \mathrm{RIU}$ \\
\hline
\end{tabular}

It should be noted that the intensity of reflection light is very weak at resonance. In order to effectively measure the resonance shift, it is necessary to increase the incident power or adopt detectors with high sensitivity. For example, assuming $10 \mathrm{~mW}$ incident light and $0.1 \mathrm{deg}$ angular resolution [25], the reflected power at the SPR dip is estimated to be $6.5 \mu \mathrm{W}$ and the reflected power variation between the resonance angle and its nearest measurable neighbor is $0.32 \mu \mathrm{W}$, which can be easily detected by commercially-available photodiodes at a visible wavelength [26]. Also note that, in our calculation, the sensing medium is set to be homogeneous in order to make fair comparison with previously works [12-16]. In fact, when the SPR biosensors are used for detecting cells with size of several microns [27], a homogeneous sensing medium layer is reasonable. To further investigate the surface sensitivity, the index change caused by the sensing target is applied with finite thickness. Under the optimal condition for $\mathrm{WS}_{2}$, the sensitivities with different sensing layer thickness are shown in Figure 3 . It is shown that the surface sensitivity increases with thicker sensing layer. Even with $10 \mathrm{~nm}$ thickness, the surface sensitivity can be still as high as $37 \mathrm{Deg} / \mathrm{RIU}$.

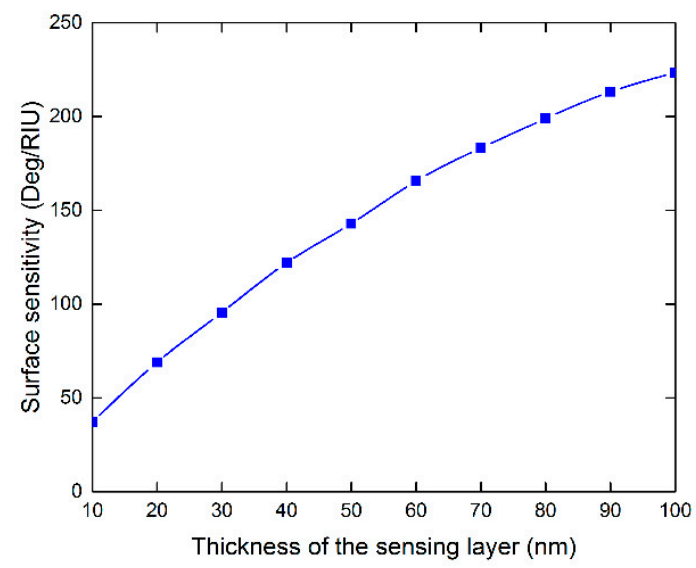

Figure 3. The change in surface sensitivity as the function of the thickness of biomolecules on the surface.

To further illustrate the contribution of $\mathrm{MX}_{2}$ on sensitivity, we have drawn the variation of the reflectance with incident angle varying from $60 \mathrm{Deg}$ to $90 \mathrm{Deg}$ for the different number of layers when the thickness of $\mathrm{Al}$ thin film is fixed at $30 \mathrm{~nm}$ and $n_{\mathrm{s}}=1.3300$ in Figure $4 \mathrm{a}-\mathrm{d}$. It is indicated that the minimum reflectivity, representing the capability of light energy absorption [15], approaches zero firstly when the number of $\mathrm{MX}_{2}$ layers increase, which means the contribution of light energy absorption exceeds the electron energy loss. Meanwhile, the full width at half maximum (FWHM) becomes broader caused by electron energy loss of $\mathrm{MX}_{2}$ layers [13]. With the further increase of the $\mathrm{MX}_{2}$ layers, the FWHM keeps getting broader and the minimum reflectivity begins to diverge from zero. This is because the SPR process must satisfy the energy conservation $T+R+A=1$, where $T, R$ and $A$ denote the transmission, reflection and absorption, respectively. Under SPR condition, since the total internal reflection is fulfilled, the transmission $T$ is close to 0 . When the number of TMDCs layers is insufficient, the absorbed light energy is not able to promote a strong SPR excitation. Therefore, increasing the TMDCs layers can enhance the light absorption, resulting in higher sensitivity. In this 
condition, the absorption $A$ is enhanced while the reflection $R$ is reduced. However, the absorption enhancement will be saturated due to the electron energy loss when further adding TMDCs layers. In this condition, the absorption $A$ is degraded while the reflection $R$ is increased.

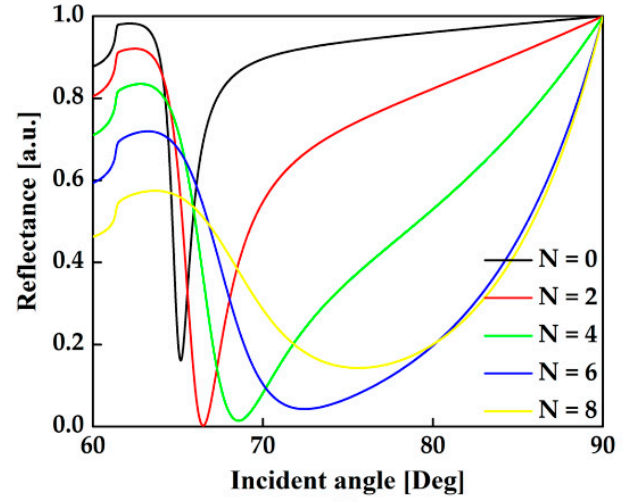

(a)

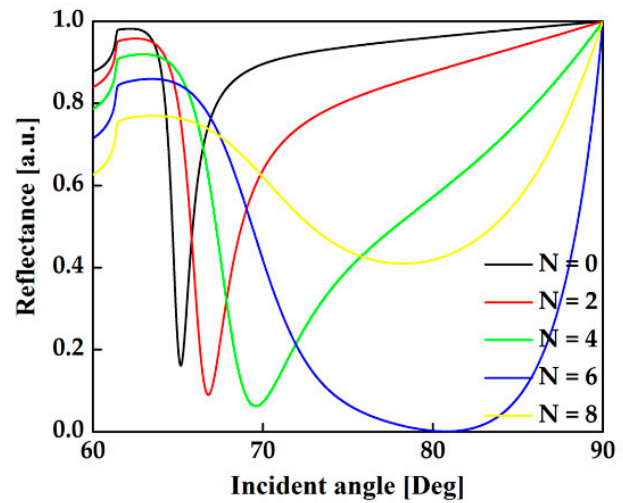

(c)

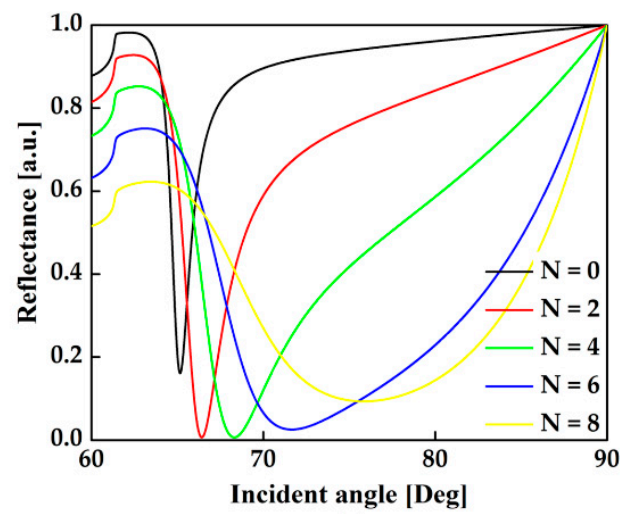

(b)

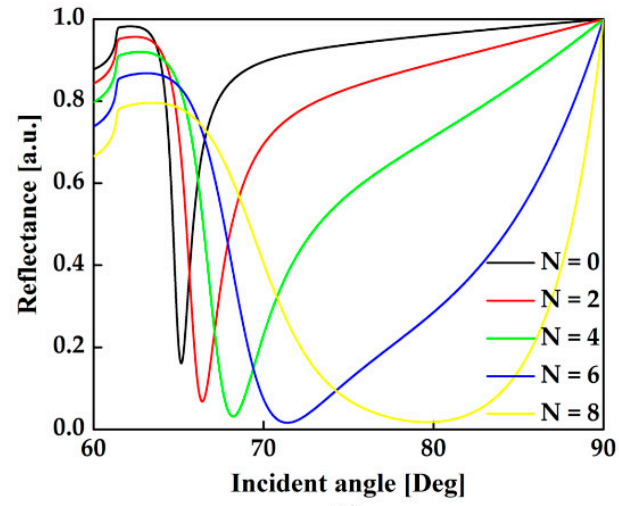

(d)

Figure 4. Variation of the reflectance respect to the different number of (a) $\mathrm{MoS}_{2}$, (b) $\mathrm{WS}_{2}$, (c) $\mathrm{MoSe}_{2}$ and (d) $\mathrm{WSe}_{2}$ layers with the thickness of $\mathrm{Al}$ thin film is fixed at $30 \mathrm{~nm}$ and the refractive index of sensing medium $\Delta n=1.3300$.

The sensitivity of conventional structure based on single Al thin film is not high enough since the metallic layer cannot absorb enough light energy to excite a strong SPR. However, TMDCs has a larger real part of dielectric constant, which benefits SPR excitation [15]. Figure 5 shows the configuration and reflectance curves for the conventional SPR sensors and the proposed one with optimal parameters. Prominent sensitivity improvement up to 3.3 times can be observed in the $\mathrm{WS}_{2}$-assisted configuration. Figure 6 plots the electric field distributions in these two structures. There is a stronger field enhancement in the $\mathrm{WS}_{2}$-assisted structure compared to the conventional one, which further verifies the positive contribution provided by the TMDCs. In addition to sensitivity, figure of merit (FOM) is also one of the important aspects that affects the sensing performance. According to our calculation, the structure without TMDCs demonstrates the highest FOM. This is because the energy loss induced by the TMDCs will broaden FWHM. As we know, this phenomenon is also reported in other works of TMDCs-based SPR sensors [12-16]. Therefore, introducing TMDCs into the SPR sensor will contribute to sensitivity enhancement but not FOM enhancement. 


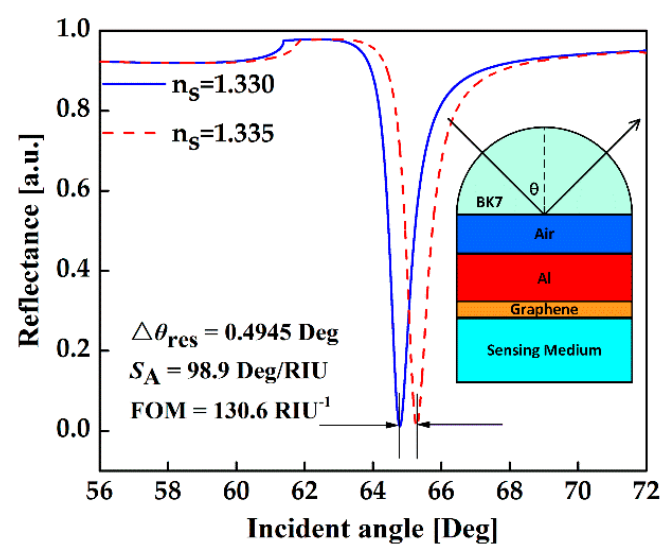

(a)

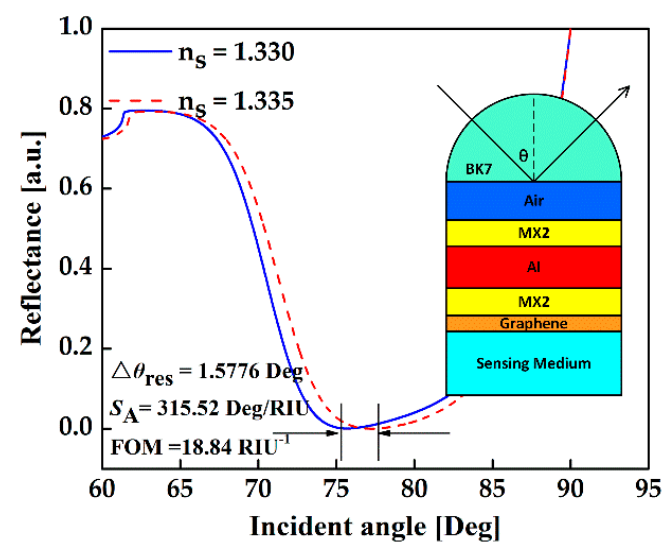

(b)

Figure 5. Variation of the reflectance with respect to the incident angle for (a) the conventional biochemical sensor based on single $\mathrm{Al}$ film, and (b) the proposed configuration based on optimal angle sensitivity.

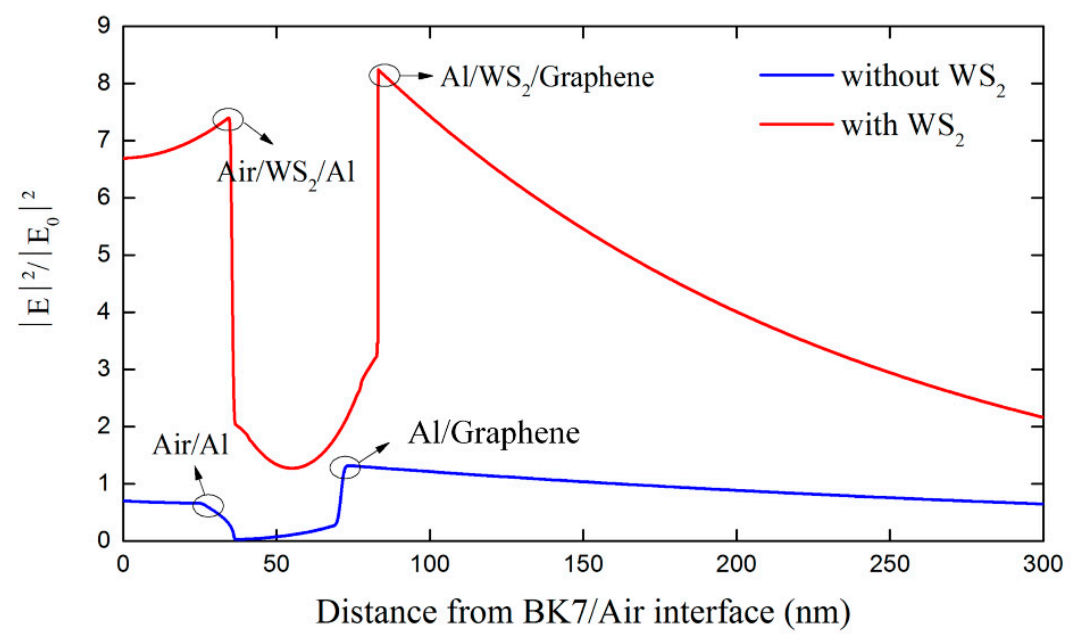

Figure 6. The field distributions along the direction perpendicular to the prism for the proposed configuration with seven $\mathrm{WS}_{2}$ layers and the conventional configuration without $\mathrm{WS}_{2}$ layers.

Besides angular sensitivity, the differential phase change between p-polarized and s-polarized reflective wave is another approach to detect the analyte [28-30]. The variation of the phase sensitivity with respect to the different number of $\mathrm{MX}_{2}$ and thickness are showed in Figure 7a-d. In the structure showing in Figure 1a, we can obtain the highest sensitivity of $1.12 \times 10^{5} \mathrm{Deg} / \mathrm{RIU}$ for bilayer $\mathrm{MoS}_{2}$ with $30 \mathrm{~nm}$ Al film, $2.02 \times 10^{5} \mathrm{Deg} / \mathrm{RIU}$ for monolayer $\mathrm{MoSe}_{2}$ with $35 \mathrm{~nm} \mathrm{Al} \mathrm{film,} 1.37 \times 10^{5} \mathrm{Deg} / \mathrm{RIU}$ for 3-layer $\mathrm{WS}_{2}$ with $35 \mathrm{~nm} \mathrm{Al} \mathrm{film} \mathrm{and} 4.56 \times 10^{5} \mathrm{Deg} / \mathrm{RIU}$ for bilayer WSe $\mathrm{W}_{2}$ with $35 \mathrm{~nm}$ Al film, respectively. Comparing with angular sensitivity, the optimal number of $\mathrm{MX}_{2}$ layers for phase sensitivity is less. 


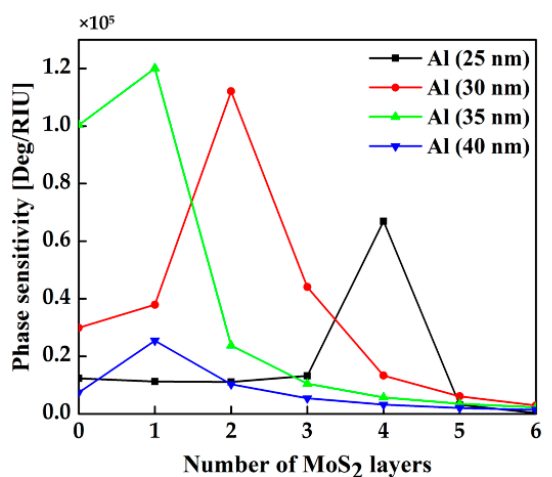

(a)

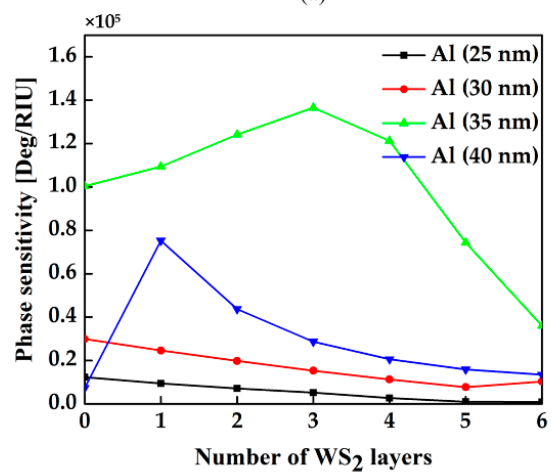

(c)

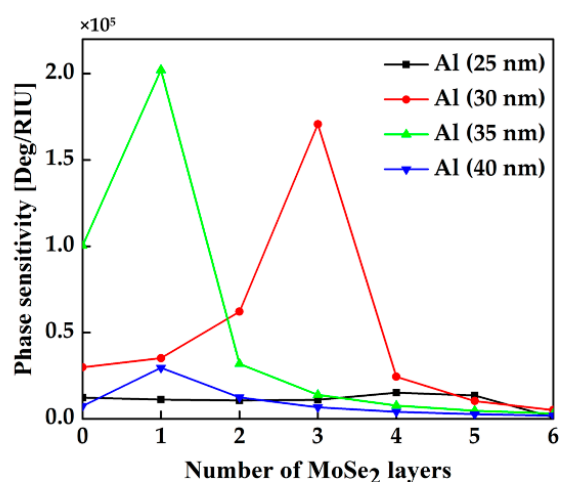

(b)

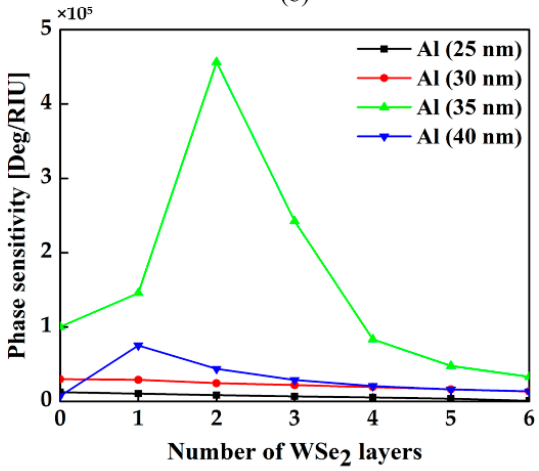

(d)

Figure 7. Variation of the angular sensitivity as a function of number of $\mathrm{MX}_{2}$ layers with various thickness of Al thin film, (a) $\mathrm{MoS}_{2}$, (b) $\mathrm{MoSe}_{2}$, (c) $\mathrm{WS}_{2}$, and (d) $\mathrm{WSe}_{2}$.

To further improve the phase sensitivity, we propose another SPR biosensor based on Kretschmann configuration, as shown in Figure $1 \mathrm{~b}$. In this sensor, Ag is used to replace $\mathrm{Al}$ and the air layer is removed. The optimal conditions of $\mathrm{Ag}$ thickness and $\mathrm{MX}_{2}$ layers are summarized in Table 3. The best phase sensitivity as high as $3.85 \times 10^{6} \mathrm{Deg} / \mathrm{RIU}$ can be achieved with monolayer $\mathrm{WS}_{2}$ and $46 \mathrm{~nm} \mathrm{Ag}$ film.

Table 3. Variation of the angular sensitivity as a function of number of $\mathrm{MX}_{2}$ layers with various thickness of $\mathrm{Al}$ thin film.

\begin{tabular}{cccc}
\hline Type of TMDC & Optimal Thickness of Al (nm) & Optimal Number of TMDC Layers & Angular Sensitivity $(\boldsymbol{\Delta} \boldsymbol{n}=\mathbf{0 . 0 0 5})$ \\
\hline $\mathrm{MoS}_{2}$ & 40 & 2 & $6.32 \times 10^{5} \mathrm{Deg} / \mathrm{RIU}$ \\
$\mathrm{MoSe}_{2}$ & 40 & 2 & $1.54 \times 10^{5} \mathrm{Deg} / \mathrm{RIU}$ \\
$\mathrm{WS}_{2}$ & 46 & 1 & $3.85 \times 10^{6} \mathrm{Deg} / \mathrm{RIU}$ \\
$\mathrm{WSe}_{2}$ & 44 & 1 & $4.57 \times 10^{5} \mathrm{Deg} / \mathrm{RIU}$ \\
\hline
\end{tabular}

For comparison, the performances of previously reported 2D-material-assisted SPR sensors are summarized in Table 4. Significant enhancements on both angular sensitivity and phase sensitivity can be obtained in the proposed sensors.

Table 4. Comparison with the formerly reported 2D-material-assisted SPR sensors.

\begin{tabular}{ccccc}
\hline 2D Material & Metal & Angular Sensitivity & Phase Sensitivity & References \\
\hline Graphene & $\mathrm{Au}$ & $134.6 \mathrm{Deg} / \mathrm{RIU}$ & - & {$[12]$} \\
Graphene and $\mathrm{MoS}_{2}$ & $\mathrm{Al}$ & $190.4 \mathrm{Deg} / \mathrm{RIU}$ & - & {$[13]$} \\
Graphene and $\mathrm{MoS}_{2}$ & $\mathrm{Au}$ & - & $8.19 \times 10^{4} \mathrm{Deg} / \mathrm{RIU}$ & {$[14]$} \\
$\mathrm{WS}_{2}$ & $\mathrm{Au}$ & $155.7 \mathrm{Deg} / \mathrm{RIU}$ & - & {$[15]$} \\
$\mathrm{WSe}_{2}$ & $\mathrm{Au}$ & - & $1.20 \times 10^{6} \mathrm{Deg} / \mathrm{RIU}$ & {$[15]$} \\
$\mathrm{BP}$ and TMDCs$/$ graphene & $\mathrm{Ag}$ & $279.0 \mathrm{Deg} / \mathrm{RIU}$ & $6.75 \times 10^{3} \mathrm{Deg} / \mathrm{RIU}$ & {$[16]$} \\
$\mathrm{WS}_{2}$ and graphene & $\mathrm{Al}$ & $315.5 \mathrm{Deg} / \mathrm{RIU}$ & & - \\
$\mathrm{WS}_{2}$ and graphene & $\mathrm{Ag}$ & - & $3.85 \times 10^{6} \mathrm{Deg} / \mathrm{RIU}$ & This work \\
\end{tabular}




\section{Conclusions}

In this paper, SPR biosensors by using 2D TMDCs are proposed to enhance the sensitivity. In such sensors, the functional materials are coated on both sides of the metal layer and the impacts of material type, layer number, and metal thickness on the sensing performance are investigated and analyzed in detail. The results show that the angular sensitivity and phase sensitivity can reach as high as 315.5 Deg/RIU with 7-layers $\mathrm{WS}_{2}$ and $3.85 \times 10^{6} \mathrm{Deg} / \mathrm{RIU}$ with 1-layers $\mathrm{WS}_{2}$, respectively. The proposed configuration can be promising a candidate for high performance biosensing.

Author Contributions: Conceptualization was designed by T.H.; methodology, validation, and formal analysis were carried out by X.Z., X.W., P.H., J.P., and Y.W.; investigation and data curation were carried out by T.H., P.S.P., and Z.C.; writing-original draft preparation was redacted by X.Z.; writing-review \& editing was performed by T.H., and X.Z.

Funding: This work was supported by the National Natural Science Foundation of China (61605179), and the Fundamental Research Funds for the Central Universities, China University of Geosciences (Wuhan) (162301132703 and G1323511794).

Conflicts of Interest: The authors declare no conflicts of interest.

\section{References}

1. Otto, A. Excitation of nonradiative surface plasma waves in silver by method of frustrated total reflection. Zeitschrift für Physik a Hadrons and Nuclei 1968, 216, 398-410. [CrossRef]

2. Kretschmann, E.; Raether, H. Notizen: Radiative decay of nonradiative surface plasmons excited by light. Zeitschrift für Naturforschung A 1968, 23, 2135-2136. [CrossRef]

3. Ahn, H.; Song, H.; Choi, J.R.; Kim, K. Localized Surface Plasmon Resonance Sensor Using DoubleMetal-Complex Nanostructures and a Review of Recent Approaches. Sensors 2018, 18, 98. [CrossRef] [PubMed]

4. Zeng, S.; Baillargeat, D.; Ho, H.P.; Yong, K.T. Nanomaterials enhanced surface plasmon resonance for biological and chemical sensing applications. Chem. Soc. Rev. 2014, 43, 3426-3452. [CrossRef] [PubMed]

5. Zeng, S.; Sreekanth, K.V.; Shang, J.; Yu, T.; Chen, C.K.; Yin, F.; Baillargeat, D.; Coquet, P.; Ho, H.P.; Kabashin, A.V.; et al. Graphene-Gold Metasurface Architectures for Ultrasensitive Plasmonic Biosensing. Adv. Mater. 2015, 27, 6163-6169. [CrossRef] [PubMed]

6. Wang, G.; Wang, C.; Yang, R.; Liu, W.; Sun, S. A Sensitive and Stable Surface Plasmon Resonance Sensor Based on Monolayer Protected Silver Film. Sensors 2017, 17, 2777. [CrossRef] [PubMed]

7. Maurya, J.B.; François, A.; Prajapati, Y.K. Two-Dimensional Layered Nanomaterial-Based One-Dimensional Photonic Crystal Refractive Index Sensor. Sensors 2018, 18, 857. [CrossRef] [PubMed]

8. Kooyman, R.P.H. Handbook of surface plasmon resonance. R. Soc. Chem. 2008, 2, 15-34.

9. Zhu, Y.; Murali, S.; Cai, W.; Li, X.; Suk, J.W.; Potts, J.R.; Ruoff, R.S. Graphene and Graphene Oxide: Synthesis, Properties, and Applications. Adv. Mater. 2010, 22, 3906-3924. [CrossRef] [PubMed]

10. Homola, J. Surface plasmon resonance sensors for detection of chemical and biological species. Chem. Rev. 2008, 108, 462-493. [CrossRef] [PubMed]

11. Zhang, N.; Humbert, G.; Gong, T.; Shum, P.P.; Li, K.; Auguste, J.L.; Wu, Z.; Hu, J.; Feng, L.; Dinh, Q.X.; et al. Side-channel photonic crystal fiber for surface enhanced Raman scattering sensing. Sens. Actuators B Chem. 2016, 233, 195-201. [CrossRef]

12. Verma, R.; Gupta, B.D.; Jha, R. Sensitivity enhancement of a surface plasmon resonance based on biomolecules sensor using graphene and silicon layers. Sens. Actuators B Chem. 2011, 160, 623-631. [CrossRef]

13. Zeng, S.; Hu, S.; Xia, J.; Anderson, T.; Dinh, X.Q.; Meng, X.M.; Coquet, P.; Yong, K.T. Graphene-MoS 2 hybrid nanostructures enhanced surface plasmon resonance biosensors. Sens. Actuators B Chem. 2015, 207, 801-810. [CrossRef]

14. Wu, L.; Jia, Y.; Jiang, L.; Guo, J.; Dai, X.; Xiang, Y.; Fan, D. Sensitivity improved SPR biosensor based on the $\mathrm{MoS}_{2}$ /graphene-aluminum hybrid structure. J. Lightwave Technol. 2016, 35, 82-87. [CrossRef]

15. Ouyang, Q.; Zeng, S.; Dinh, X.Q.; Coquet, P.; Yong, K.T. Sensitivity enhancement of MoS $_{2}$ nanosheet based surface Plasmon resonance biosensor. Procedia Eng. 2016, 140, 134-139. [CrossRef] 
16. Wu, L.; Guo, J.; Wang, Q.; Lu, S.; Dai, X.; Xiang, Y.; Fan, D. Sensitivity enhancement by using few-layer black phosphorus-graphene/TMDCs heterostructure in surface plasmon resonance biochemical sensor. Sens. Actuators B. Chem. 2017, 249, 542-548. [CrossRef]

17. Khageswar, S.; Kumar, M.S.; Kumar, G.P. He-Ne laser $(632.8 \mathrm{~nm})$ pre-irradiation gives protection against DNA damage induced by a near-infrared trapping beam. J. Biophotonics 2009, 2, 140-144.

18. Sreekanth, K.V.; Zeng, S.; Yong, K.T.; Yu, T. Sensitivity enhanced biosensor using graphene-based one-dimensional photonic crystal. Sens. Actuators B. Chem. 2013, 182, 424-428. [CrossRef]

19. Jha, R.; Sharma, A.K. Chalcogenide glass prism based SPR sensor with Ag-Au bimetallic nanoparticle alloy in infrared wavelength region. J. Opt. Pure Appl. Opt. 2009, 11, 045502. [CrossRef]

20. Li, Y.; Chernikov, A.; Zhang, X.; Rigosi, A.; Hill, H.M.; van der Zande, A.M.; Chenet, D.A.; Shih, E.M.; Hone, J.; Heinz, T.F. Measurement of the optical dielectric function of monolayer transition-metal dichalcogenides: $\mathrm{MoS}_{2}, \mathrm{MoSe}_{2}, \mathrm{WS}_{2}$, and WSe 2 . Phys. Rev. B 2014, 90, 205-422. [CrossRef]

21. Liu, H.L. Optical properties of monolayer transition metal dichalcogenides probed by spectroscopic ellipsometry. Appl. Phys. Lett. 2014, 105, 201905. [CrossRef]

22. Bruna, M.; Borini, S. Optical constants of graphene layers in the visible range. Appl. Phys. Lett. 2009, 94, 031901. [CrossRef]

23. Wu, L.; Ling, Z.; Jiang, L.; Guo, J.; Dai, X.; Xiang, Y.; Fan, D. Long-Range Surface Plasmon with Graphene for Enhancing the Sensitivity and Detection Accuracy of Biosensor. IEEE Photonics J. 2016, 8, 1-9. [CrossRef]

24. Maharana, P.K.; Jha, R.; Palei, S. Sensitivity enhancement by air mediated graphene multilayer based surface plasmon resonance biosensor for near infrared. Sens. Actuators B Chem. 2014, 190, 494-501. [CrossRef]

25. Sreekanth, K.V.; Alapan, Y.; ElKabbash, M.; Wen, A.M.; Ilker, E.; Hinczewski, M.; Gurkan, U.A.; Steinmetz, N.F.; Strangi, G. Enhancing the Angular Sensitivity of Plasmonic Sensors Using Hyperbolic Metamaterials. Adv. Opt. Mater. 2016, 4, 1767-1772. [CrossRef] [PubMed]

26. Corcoran, B.; Monat, C.; Grillet, C.; Moss, D.J.; Eggleton, B.J.; White, T.P.; O'Faolain, L.; Krauss, T.F. Green light emission in silicon through slow-light enhanced third-harmonic generation in photonic-crystal waveguidese. Nat. Photonics 2009, 3, 206-210. [CrossRef]

27. Song, C.L.; Jin, T.; Yan, R.P.; Qi, W.Z.; Huang, T.Y.; Ding, H.F.; Tan, S.H.; Nguyen, N.T.; Xi, L. Opto-acousto-fluidic microscopy for three-dimensional label-free detection of droplets and cells in microchannels. Lab Chip 2018, 9, 1267-1390. [CrossRef] [PubMed]

28. Zeng, S.; Yu, X.; Law, W.C.; Zhang, Y.; Hu, R.; Dinh, X.Q.; Ho, H.P.; Yong, K.T. Size dependence of Au NP-enhanced surface plasmon resonance based on differential phase measurement. Sens. Actuators B Chem. 2013, 176, 1128-1133. [CrossRef]

29. Raether, H. Surface plasmons on smooth and rough surfaces and on gratings. Springer Tracts Mod. Phys. 1983, 111, 354401-373633.

30. Wong, C.L.; Chua, M.; Mittman, H.; Choo, L.X.; Lim, H.Q.; Olivo, M. A Phase-Intensity Surface Plasmon Resonance Biosensor for Avian Influenza A (H5N1) Detection. Sensors 2017, 17, 2363. [CrossRef] [PubMed]

(C) 2018 by the authors. Licensee MDPI, Basel, Switzerland. This article is an open access article distributed under the terms and conditions of the Creative Commons Attribution (CC BY) license (http://creativecommons.org/licenses/by/4.0/). 